\title{
1 Urine metabolomics of rats with chronic atrophic gastritis
}

2 Urine metabolomics

3 Guo-Xiu Zu ${ }^{1 \pi}$, Qian-Qian Sun ${ }^{1 \pi}$, Jian-Chen ${ }^{1,4 \pi}$, Xi-Jian Liu ${ }^{1}$, Ke-Yun Sun ${ }^{1}$, Liang-Kun Zhang ${ }^{1}$,

4 Ling li $^{1}$, Tao Han $^{2 *}$, Ha I-Liang Huang ${ }^{3 *}$

5

$6 \quad{ }^{1}$ Department of Traditional Chinese Medicine, Shandong University of Traditional Chinese

7 Medicine, Jinan, Shandong, China

$8{ }^{2}$ Graduate Office ,Shandong University of Traditional Chinese Medicine, Jinan, Shandong,

9 China

$10{ }^{3}$ Department of Rehabilitation Medicine, Shandong University of Traditional Chinese Medicine,

11 Jinan, Shandong, China

$12{ }^{4}$ Affiliated Central Hospital of Shandong First Medical University, Shandong First Medical

13 University, Jinan, Shandong, China

14 I These authors contributed equally to this work.

15

$16 *$ Corresponding author

17 E-mail: 60012002@sdutcm.edu.cn (TH)

18 E-mail: 06000031@sdutcm.edu.cn (HH)

19 


\section{Abstract}

21 Background/Aim: To use liquid chromatography-mass spectrometry (LC-MS) to identify

22 endogenous differential metabolites in the urine of rats with chronic atrophic gastritis (CAG).

23 Materials and Methods: Methylnitronitrosoguanidine (MNNG) was used to produce a CAG model

24 in Wistar rats, and HE staining was used to determine the pathological model. LC-MS was used

25 to detect the differential metabolic profiles in rat urine. Diversified analysis was performed by the

26 statistical method. Results: Compared with the control group, the model group had 68 differential

27 metabolites, 25 that were upregulated and 43 that were downregulated. The main metabolic

28 pathways were D-glutamine and D-glutamic acid metabolism, histidine metabolism and purine

29 metabolism. Conclusion: By searching for differential metabolites and metabolic pathways in the

30 urine of CAG rats, this study provides effective experimental data for the pathogenesis and clinical

31 diagnosis of CAG. 


\section{Introduction}

Chronic atrophic gastritis (CAG) is a type of atrophy of gastric mucosal epithelial cells and

36 glands where the number of glands is reduced, the mucosal layer thins, and the mucosal muscle

37 layer thickens and may be accompanied by intestinal metaplasia and dysplasia. Digestive system

38 diseases [1] mainly have the clinical manifestations of bloating, fullness of the stomach, belching,

39 pain in the upper abdomen, loss of appetite, weight loss, etc. CAG has a wide variety of factors

40 and is a common and frequently occurring clinical disease with a $2.55 \%-7.46 \%$ canceration rate

41 [2]. In 1978, the World Health Organization officially defined chronic atrophic gastritis as a

42 precancerous state. The active treatment of CAG in clinical practice is an important node to block

43 its development into gastric cancer.

44 As an important branch of systems biology, metabolomics technology is unique because it

45 does not require the establishment of a large database of expressed gene sequences [3].

46 Metabolomics can express the physiological and biochemical state of the body through biological

47 metabolic structure to better analyze pathogenesis. Among its advantages, liquid chromatography-

48 mass spectrometry (LC-MS) technology can be directly used to analyze biological metabolites to

49 obtain final analysis results with the advantage of finding subtle changes in gene and protein

50 expression during biological metabolism. Thus, LC-MS has become the most commonly used

51 analytical technique in metabolomics research [4]. This study explains the molecular mechanism

52 of action and metabolic pathways of chronic atrophic gastritis through pharmacodynamics and LC-

53 MS. 


\section{Materials and methods}

\section{Animals}

Twenty SPF grade Wistar male rats, 6 weeks old, $180 \pm 20$ g, were provided by Shandong

59 Pengyue Experimental Animal Co., Ltd. [SCXK (Lu) 20140007]. The feeding environment was a

60 temperature of $26^{\circ} \mathrm{C} \pm 2{ }^{\circ} \mathrm{C}$, humidity $50 \pm 10 \%$, and light illumination/dark cycle $12 \mathrm{~h}$. The

61 experiment started after $7 \mathrm{~d}$ of adaptive feeding from the time of purchase. During the period, the

62 animals has free access to food and drinking water, and the experiment met animal ethical

63 requirements.

\section{Experimental reagents and instruments}

Methylnitronitrosoguanidine (manufactured by Tokyo Chemical Industry Co., Ltd.,

67 NH8JH-DR), vetzyme tablets (Lepu Hengjiuyuan Pharmaceutical Co., Ltd., 20170401), ranitidine

68 hydrochloride capsules (Tianjin Pacific Pharmaceutical Co., Ltd., 20170601), and ammonium

69 hydroxide (Shanghai Wokai Biotechnology Co., Ltd., 20170220) were used. Anhydrous ethanol

70 (Tianjin Fuyu Fine Chemical Co., Ltd., 20170808), methanol (Woke), acetonitrile and formic acid

71 (Aladdin), ammonium formate (Sigma), hematoxylin staining solution, eosin staining solution,

72 differentiation solution, blue back solution (Hebei Bohai Biological Engineering Development 
73 Co., Ltd.), and xylene (Tianjin Yongda Chemical Reagent Co., Ltd.) were also utilized in this

74 study.

A refrigerated centrifuge (Eppendorf, H1650-W), mixer (Vortex Mixer, QL-866), liquid chromatograph (Thermo, UltiMate 3000) and mass spectrometer Thermo (Q Exactive Focus) were

77 instruments used in this study.

79 Animal model

Twenty Wistar rats were prepared, and 10 rats were randomly selected as a blank group.

81 Normal diet was fed until the materials were collected. The remaining rats were model rats

82 according to the following method [5]: rats were given $120 \mu \mathrm{g} / \mathrm{mL} \mathrm{MNNG}$ from the 1 st day of

83 modeling, given $0.1 \%$ ammonia water freely for $24 \mathrm{~h}$ fed with $0.03 \%$ ranitidine feed using the

84 hunger and satiety method (full food for $2 \mathrm{~d}$, fasting for $1 \mathrm{~d}$ ), an each given $2 \mathrm{ml}$ of $40 \%$ ethanol

85 on the fasting day. The above operation lasted for 16 weeks and each rat was weighed twice a

86 week during the modeling process. During the experiment, the weight, coat color and behavior of

87 the rats were observed.

\section{Urine collection and preparation}

90 Before taking the material, the rats were fasted for $24 \mathrm{~h}$ while drinking water normally, and

91 urine was collected from the rats. Urine was centrifuged at $2500 \mathrm{rpm}$ at room temperature for 1

92 hour in the morning, and the supernatant was divided into centrifuge tubes; each tube was $>0.3$ 
$93 \mathrm{ml}$. The urine samples were melted at $4{ }^{\circ} \mathrm{C}$ and $100 \mu \mathrm{L}$ of each sample was placed into a $1.5 \mathrm{~mL}$

94 centrifuge tube, $100 \mu \mathrm{L}$ of $\mathrm{ddH}_{2} \mathrm{O}$ was added followed by shaking for 5 min to fully absorb and

95 centrifugation at $10000 \mathrm{~g}$ and $4{ }^{\circ} \mathrm{C}$ for $10 \mathrm{~min}$. Then, a $0.22 \mu \mathrm{m}$ membrane was used to filter the

96 supernatant to obtain the samples to be tested; $20 \mu \mathrm{L}$ of the synthetic QC samples were extracted

97 from each sample to be tested, and the remaining samples were tested by LC-MS.

98

99 LC-MS chromatographic mass spectrometry conditions

100

A Thermo Ultimate 3000 chromatograph and an ACQUITY UPLC® HSS T3 $1.8 \mu \mathrm{m}(2.1$

$101 \times 150 \mathrm{~mm}$ ) chromatographic column were used with an autosampler temperature of $8{ }^{\circ} \mathrm{C}$, a flow

102 rate of $0.25 \mathrm{~mL} / \mathrm{min}$, and a column temperature of $40{ }^{\circ} \mathrm{C}$. The sample was eluted with an injection

103 volume of $2 \mu \mathrm{l}$, and the positive mode mobile phases were $0.1 \%$ formic acid in water (A) and $0.1 \%$

104 formic acid in acetonitrile (B). The gradient elution program was $0 \sim 2 \mathrm{~min}, 2 \% \mathrm{~B} ; 2 \sim 10 \mathrm{~min}$,

$1052 \% \sim 50 \% \mathrm{~B} ; 10 \sim 15 \min , 50 \% \sim 98 \% \mathrm{~B} ; 15 \sim 20 \min , 98 \% \mathrm{~B} ; 20 \sim 22 \min , 98 \% \sim 2 \% \mathrm{~B}$

$10622 \sim 25 \mathrm{~min}, 2 \% \mathrm{~B}$. The negative mode mobile phases were $5 \mathrm{mM}$ ammonium formate $(\mathrm{A})$ and

107 acetonitrile (B). The gradient elution program was $0 \sim 2 \min , 2 \% \mathrm{~B} ; 2 \sim 10 \mathrm{~min}, 2 \% \sim 50 \% \mathrm{~B}$;

$10810 \sim 15 \min , 50 \% \sim 98 \% \mathrm{~B} ; 15 \sim 20 \mathrm{~min}, 98 \% \mathrm{~B} ; 20 \sim 22 \mathrm{~min}, 98 \% \sim 2 \% \mathrm{~B} ; 22 \sim 25 \min$

$1092 \%$ B [6]. The Thermo Q Exactive Focus mass spectrometer was operated with the following

110 conditions: electrospray ion (ESI) source, positive and negative ion ionization mode, positive ion

111 spray voltage of $3.50 \mathrm{kV}$, negative ion spray voltage of $-2.50 \mathrm{kV}$, sheath gas of 30 arb, auxiliary

112 gas of $10 \mathrm{arb}$, capillary temperature of $325^{\circ} \mathrm{C}$, full scan with a resolution of 70,000 , scan range of 
$113 \mathrm{~m} / \mathrm{z}$ 81-1000, secondary cracking with $\mathrm{HCD}$, collision voltage of $30 \mathrm{eV}$, and dynamic exclusion to

114 remove unnecessary MS/MS information.

115

\section{Data processing}

117 The obtained raw data was converted to mzXML format with ProteoWizard software

118 (v3.0.8789) [7] and the RCMS (v3.3.2) XCMS package was used for peak identification, peak

119 filtering, and peak alignment analysis. The main parameters were $b w=5, p p m=15$, peakwidth $=$

$120 \mathrm{c}(10,20)$, mzwid $=0.015$, mzdiff $=0.01$, and method $=$ centWave, which includes the mass to

121 charge ratio $(\mathrm{m} / \mathrm{z})$ and information data matrix such as retention time (rt) and intensity.

\section{Results}

\section{General situation}

126 Control group: In good condition, sturdy body, strong limbs, neat, supple and shiny fur, mental

127 state is excellent, responsive to external conditions, body weight gradually increases, and the stool

128 is normal. Model group: Poor condition, thin body, weak limbs, messy fur, dryness, and dullness,

129 poor mental state, drowsiness, unresponsive to external conditions, insignificant changes in body

130 mass, slower rise, and less stool that is hard. Body mass changes are shown in Fig. 1.

\section{Fig. 1. Mass Variation Diagram of the Control Group and Model Group.}




\section{Observation of pathological tissues}

134 As shown in Fig. 2A, the gastric tissue mucosa lamina propria in the blank group pathological

135 section is rich in gastric glands that are closely arranged with a normal structure, and the gastric

136 gland epithelial cells have a normal morphology. In the model group, the lamina propria were

137 loosely arranged, the lamina propria of the gastric mucosa was severely congested (black arrow),

138 and there were a large number of inflammatory cells (blue arrow) under the mucosa with edema,

139 as shown in Fig. 2B.

140 Fig. 2. HE Staining Pathological Sections. A. HE Staining Pathological Sections of Gastric

141 Mucosa in the Control Group (×200); 2-B: HE Staining Pathological Sections of Gastric Mucosa

142 in the Model Group (×200).

\section{Chromatogram in total ion mode:}

145 The components separated by chromatography entered into mass spectrometry (MS)

146 analysis, and data collection was performed by continuous scanning of the mass spectrum. The

147 intensity is on the ordinate, and the time is on the abscissa. The resulting spectrum is the base peak

148 chromatogram (BPC); see Fig. 3A and B (G: model group, H: control group)

149 Fig. 3. Chromatogram in Total Ion Mode. 3-A: Typical Sample BPC in Positive Ion Mode, 3- 
164 of the data model, a permutation test was performed on the model (Fig. 4D). The above results

165 show that the multivariate data model of urine samples meets the parameter standard, indicating

166 that the model has high stability and good predictive ability.

167 Fig. 4. Urine Metabolism Profile of CAG Model Rats in Positive Ion Mode. 4-A: PCA Scores,

\section{Analysis of urine metabolomics in negative ion mode}


174 between groups were obtained from the score chart. The PCA score graph shows that most samples

175 fall within the ellipse of the $95 \%$ confidence interval, with only a few outliers. The PCA score

176 (Fig. 5A) graph shows the spatial distribution of the urine samples of the two groups, which can

177 be significantly separated. PLS-DA and OPLS-DA analysis methods were used to further analyze

178 the full spectrum of urine, and the results showed that the two groups of samples could be

179 significantly separated (Fig. 5B and C). In order to test whether the repeatability of the model is

180 good and to ensure reliability of the data model, the model was replaced and verified (Fig. 5D).

181 The intercept of Q2 is negative, indicating that the model is valid. The above results indicate that

182 the multivariate data model of urine samples meets the parameter standard, indicating that the

183 model has high stability and good predictive ability.

184 Fig. 5. Urine Metabolism Profile of CAG Model Rats in Negative Ion Mode. 5-A: PCA Scores,

185 5-B: PLS-DA Scores, 5-C: OPLS-DA Scores, 5-D: Replacement Test of the CAG Model Urine

186 Fit Model in Negative Ion Mode.

\section{Extraction and analysis of differential metabolites}

From the PCA, PLS-DA, OPLS-DA analysis model group and blank group, the screening

190 conditions were in accordance with a P-value $\leq 0.05$, VIP $\geq 1$ [6], and molecular weight error $<20$

$191 \mathrm{ppm})$. According to the fragmentation information obtained from MS/MS mode, further matching 
192 annotations were obtained in the HMDB, METLIN, MassBank, LipidMaps, and mzCloud

193 databases to obtain accurate metabolite information. A total of 68 differential metabolites were

194 screened, of which 25 were upregulated and 43 that were downregulated, compared with

195 metabolites with the same or similar metabolic modes clustered to obtain differential metabolite

196 heat maps and metabolite correlation heat maps (Fig. 6). These differential metabolites relied on

197 the Marker-view, KEGG, HMDB, MetaboAnalyst and other databases, which were searched and

198 identified, and the results are shown in Table 1.

199 Fig. 6. Heat Map of the Differential Metabolites. A: Heat Map of the Differential Metabolites

200 in CAG Rats; 6-B: Correlation Heat Map of Differential Metabolites in CAG Rats.

201 Table 1. Differential Metabolic Markers in Urine of CAG Rats (Upregulated $\uparrow$,

202 Downregulated $\downarrow$ ).

\begin{tabular}{|c|c|c|c|c|}
\hline chemical compound & chemical formula & del vs Control_ & $\log 2(\mathrm{FC})$ & $\mathrm{p}$ value \\
\hline Arbutin & $\mathrm{C} 12 \mathrm{H} 16 \mathrm{O} 7$ & 1.771574784 & 2.3461 & $0.012648447 \uparrow$ \\
\hline Inosine & $\mathrm{C} 10 \mathrm{H} 12 \mathrm{~N} 4 \mathrm{O} 5$ & 1.528975498 & 2.1558 & $0.037258488 \uparrow$ \\
\hline Adenosine & $\mathrm{C} 10 \mathrm{H} 13 \mathrm{~N} 5 \mathrm{O} 4$ & 1.934286006 & 2.0713 & $0.004285334 \uparrow$ \\
\hline 5-S-Methyl-5-thioadenosine & $\mathrm{C} 11 \mathrm{H} 15 \mathrm{~N} 5 \mathrm{O} 3 \mathrm{~S}$ & 2.284849647 & 1.8528 & $0.000234115 \uparrow$ \\
\hline 10-Hydroxy capric acid & $\mathrm{C} 10 \mathrm{H} 20 \mathrm{O} 3$ & 1.823597606 & 1.5684 & $0.008306808 \uparrow$ \\
\hline 7-Methylguanosine & C11H16N5O5 & 1.568140916 & 1.549 & $0.031843615 \uparrow$ \\
\hline Pipecolic acid & C6H11NO2 & 1.781759339 & 1.5181 & $0.010438908 \uparrow$ \\
\hline delta-Decalactone & $\mathrm{C} 10 \mathrm{H} 18 \mathrm{O} 2$ & 1.849847045 & 1.5177 & $0.007156206 \uparrow$ \\
\hline
\end{tabular}




\begin{tabular}{|c|c|c|c|c|}
\hline 3-Methyl-L-histidine & $\mathrm{C} 7 \mathrm{H} 11 \mathrm{~N} 3 \mathrm{O} 2$ & 1.872481394 & 1.4212 & $0.00738418 \uparrow$ \\
\hline Dihydro-3-coumaric acid & $\mathrm{C} 9 \mathrm{H} 10 \mathrm{O} 3$ & 1.528214666 & 1.394 & $0.037370134 \uparrow$ \\
\hline Adipic acid & C6H10O4 & 2.113128751 & 1.3866 & $0.001516902 \uparrow$ \\
\hline 2-Methylguanosine & C11H15N5O5 & 1.751775025 & 1.367 & $0.012215874 \uparrow$ \\
\hline Guanosine & C10H13N5O5 & 1.53671651 & 1.3507 & $0.032900583 \uparrow$ \\
\hline Suberic acid & C8H14O4 & 1.612701672 & 1.3484 & $0.026434225 \uparrow$ \\
\hline 2-Deoxycytidine & $\mathrm{C} 9 \mathrm{H} 13 \mathrm{~N} 3 \mathrm{O} 4$ & 1.915004809 & 1.3307 & $0.004840515 \uparrow$ \\
\hline D-Glucuronic acid & C6H10O7 & 2.241280415 & 1.2756 & $0.000512579 \uparrow$ \\
\hline CMPF & $\mathrm{C} 12 \mathrm{H} 16 \mathrm{O} 5$ & 2.124552651 & 1.2416 & $0.001388456 \uparrow$ \\
\hline Gentisic acid & $\mathrm{C} 7 \mathrm{H} 6 \mathrm{O} 4$ & 1.835909917 & 1.2406 & $0.009038573 \uparrow$ \\
\hline TMCA & C12H14O5 & 1.954028191 & 1.161 & $0.003771041 \uparrow$ \\
\hline 2-Aminopteridine-4,7-Diol & C6H5N5O2 & 2.016289866 & 1.1144 & $0.003043425 \uparrow$ \\
\hline 4-Hydroxy nonenal Mercapturic acid & $\mathrm{C} 14 \mathrm{H} 25 \mathrm{NO} 5 \mathrm{~S}$ & 1.548812817 & 1.0965 & $0.034435408 \uparrow$ \\
\hline D-Biotin & C10H16N2O3S & 1.515098427 & 1.0857 & $0.035928770 \uparrow$ \\
\hline N-Acetylcadaverine & $\mathrm{C} 7 \mathrm{H} 16 \mathrm{~N} 2 \mathrm{O}$ & 1.640234776 & 1.0602 & $0.020995681 \uparrow$ \\
\hline Benzaldehyde & $\mathrm{C} 7 \mathrm{H} 6 \mathrm{O}$ & 1.762739968 & 1.0398 & $0.011540491 \uparrow$ \\
\hline Guanidinoacetic acid & $\mathrm{C} 3 \mathrm{H} 7 \mathrm{~N} 3 \mathrm{O} 2$ & 1.705152566 & 1.0027 & $0.015441198 \uparrow$ \\
\hline 3-Methylindole & C9H9N & 1.876426797 & 0.9857 & $0.007221167 \downarrow$ \\
\hline 2-Oxoglutaric acid & C5H6O5 & 1.495118055 & 0.9552 & $0.042475723 \downarrow$ \\
\hline Marmesin acetate & $\mathrm{C} 16 \mathrm{H} 16 \mathrm{O} 5$ & 1.720421852 & 0.9461 & $0.014319362 \downarrow$ \\
\hline
\end{tabular}




\begin{tabular}{|c|c|c|c|c|}
\hline N-Lactoyl-phenylalanine & $\mathrm{C} 12 \mathrm{H} 15 \mathrm{NO} 4$ & 1.57132921 & 0.907 & $0.028460454 \downarrow$ \\
\hline Taxifolin & $\mathrm{C} 15 \mathrm{H} 12 \mathrm{O} 7$ & 1.718717369 & 0.8785 & $0.016387198 \downarrow$ \\
\hline 3-(3,4-Dihydroxyphenyl)Propanoic acid & C9H10O4 & 2.070984986 & 0.8642 & $0.002077192 \downarrow$ \\
\hline $\mathrm{N}(2)$-Acetyl-L-Lysine & $\mathrm{C} 8 \mathrm{H} 16 \mathrm{~N} 2 \mathrm{O} 3$ & 2.049816081 & 0.8419 & $0.001930407 \downarrow$ \\
\hline L-Phenylalanyl-L-Proline & $\mathrm{C} 14 \mathrm{H} 18 \mathrm{~N} 2 \mathrm{O} 3$ & 2.077282892 & 0.7931 & $0.001566615 \downarrow$ \\
\hline O-Toluic acid & $\mathrm{C} 8 \mathrm{H} 8 \mathrm{O} 2$ & 1.805758215 & 0.7836 & $0.009169037 \downarrow$ \\
\hline Quinaldic acid & $\mathrm{C} 10 \mathrm{H} 7 \mathrm{NO} 2$ & 1.669168777 & 0.7797 & $0.020624237 \downarrow$ \\
\hline 3-Hydroxy-3-methylglutaric acid & C6H10O5 & 1.480741362 & 0.7542 & $0.044849483 \downarrow$ \\
\hline Dopamine & $\mathrm{C} 8 \mathrm{H} 11 \mathrm{NO} 2$ & 1.825498392 & 0.753 & $0.008218865 \downarrow$ \\
\hline Syringic acid & $\mathrm{C} 9 \mathrm{H} 10 \mathrm{O} 5$ & 1.538159222 & 0.7057 & $0.035930624 \downarrow$ \\
\hline N-Acetyl-Glutamic acid & C7H11NO5 & 1.606367357 & 0.6886 & $0.024447196 \downarrow$ \\
\hline N,N-Diethyl-M-Toluamide & $\mathrm{C} 12 \mathrm{H} 17 \mathrm{NO}$ & 1.658473782 & 0.6825 & $0.019299997 \downarrow$ \\
\hline N-epsilon-Acetyl-L-lysine & $\mathrm{C} 8 \mathrm{H} 16 \mathrm{~N} 2 \mathrm{O} 3$ & 2.024611789 & 0.6354 & $0.00287668 \downarrow$ \\
\hline Glutaric acid & C5H8O4 & 1.55606035 & 0.6234 & $0.033445496 \downarrow$ \\
\hline 3-Indoleacetic acid & $\mathrm{C} 10 \mathrm{H} 9 \mathrm{NO} 2$ & 1.490647745 & 0.5842 & $0.043203485 \downarrow$ \\
\hline Homovanillic acid & C9H10O4 & 1.602561332 & 0.5841 & $0.027598265 \downarrow$ \\
\hline L-Histidine & C6H9N3O2 & 1.603838417 & 0.5723 & $0.024721392 \downarrow$ \\
\hline Hexanoylcarnitine & $\mathrm{C} 13 \mathrm{H} 25 \mathrm{NO} 4$ & 1.466692106 & 0.5425 & $0.043469353 \downarrow$ \\
\hline Nonic acid & C9H16O4 & 2.208499912 & 0.5276 & $0.000690754 \downarrow$ \\
\hline 4-Acetamidobutanoic acid & C6H11NO3 & 1.641325837 & 0.526 & $0.02089112 \downarrow$ \\
\hline
\end{tabular}




\begin{tabular}{|c|c|c|c|c|}
\hline N-Acetyl-beta-Alaninate & C5H9NO3 & 1.573650573 & 0.5139 & $0.028179966 \downarrow$ \\
\hline (S)-2-Hydroxyglutarate & $\mathrm{C} 5 \mathrm{H} 8 \mathrm{O} 5$ & 1.464880663 & 0.5013 & $0.047581863 \downarrow$ \\
\hline N-Acetyl-L-Histidine & $\mathrm{C} 8 \mathrm{H} 11 \mathrm{~N} 3 \mathrm{O} 3$ & 1.573531368 & 0.4979 & $0.028194318 \downarrow$ \\
\hline 2-Pyrrolidone-5-Carboxylic acid, Methyl Ester & C6H9NO3 & 1.868632508 & 0.4193 & $0.006413493 \downarrow$ \\
\hline 2-Hydroxypropanoic acid & $\mathrm{C} 3 \mathrm{H} 6 \mathrm{O} 3$ & 1.874058772 & 0.3997 & $0.00731866 \downarrow$ \\
\hline 3-Hydroxycapric acid & $\mathrm{C} 10 \mathrm{H} 20 \mathrm{O} 3$ & 1.944065438 & 0.3836 & $0.004843594 \downarrow$ \\
\hline (R-Noradrenaline & $\mathrm{C} 8 \mathrm{H} 11 \mathrm{NO} 3$ & 1.526685758 & 0.3797 & $0.037595251 \downarrow$ \\
\hline Dacarbazine & C6H10N6O & 1.587732534 & 0.2827 & $0.026523445 \downarrow$ \\
\hline Phenacylamine & $\mathrm{C} 8 \mathrm{H} 9 \mathrm{NO}$ & 1.489849766 & -0.4527 & $0.039727046 \downarrow$ \\
\hline Threonate & $\mathrm{C} 4 \mathrm{H} 8 \mathrm{O} 5$ & 1.547635686 & -0.6082 & $0.034598259 \downarrow$ \\
\hline Adenine & C5H5N5 & 1.792987662 & -0.7032 & $0.011340028 \downarrow$ \\
\hline Dodecanedioic acid & $\mathrm{C} 12 \mathrm{H} 22 \mathrm{O} 4$ & 1.66119894 & -0.9546 & $0.021378107 \downarrow$ \\
\hline 2-Deoxyuridine & $\mathrm{C} 9 \mathrm{H} 12 \mathrm{~N} 2 \mathrm{O} 5$ & 1.943477192 & -0.979 & $0.004861136 \downarrow$ \\
\hline Formononetin & $\mathrm{C} 16 \mathrm{H} 12 \mathrm{O} 4$ & 2.025071834 & -1.1042 & $0.002867682 \downarrow$ \\
\hline L-Glutamic acid & $\mathrm{C} 5 \mathrm{H} 9 \mathrm{NO} 4$ & 1.777690879 & -1.1167 & $0.010667276 \downarrow$ \\
\hline Genkwanin & $\mathrm{C} 16 \mathrm{H} 12 \mathrm{O} 5$ & 2.062070851 & -1.1857 & $0.002214871 \downarrow$ \\
\hline Nicotinic acid & $\mathrm{C} 6 \mathrm{H} 5 \mathrm{NO} 2$ & 2.168087639 & -1.2801 & $0.000737259 \downarrow$ \\
\hline Guanine & $\mathrm{C} 5 \mathrm{H} 5 \mathrm{~N} 5 \mathrm{O}$ & 2.476437968 & -1.3203 & $3.25 \mathrm{E}-05 \downarrow$ \\
\hline Adenosine 3-monophosphate & C10H14N5O7P & 1.68169368 & -1.3227 & $0.019481197 \downarrow$ \\
\hline AMP & C10H14N5O7P & 1.900260783 & -1.5778 & $0.005302741 \downarrow$ \\
\hline
\end{tabular}




\section{CAG model group urine differential metabolite pathway information}

210 metabolism, niacin-nicotinamide metabolism, pentose-glucuronate interconversion, pyrimidine

211 metabolism, lysine degradation, citric acid cycle (TCA cycle), starch-sucrose metabolism, Inositol

212 phosphate metabolism, glutathione metabolism, porphyrin and chlorophyll metabolism, cysteine-

213 methionine metabolism, glycine-serine-threonine metabolism, aminoacyl-tRNA biosynthesis, and

214 tryptophan metabolism. Among them, the metabolic pathways with $* \mathrm{P}<0.5$ and Impact $>0$ include

215 D-glutamine and D-glutamic acid metabolism, histidine metabolism, and purine metabolism, as

216 shown in Table 2.

217 Fig. 7. Metabolic Pathways of CAG Rat Metabolites Mapped to KEGG.

218 Table 2. Differential Metabolic Pathways in Urine of CAG Rats

\begin{tabular}{|c|c|c|}
\hline D-Glutamine and $\mathrm{D}^{-}$ & Histidine & Purine metabolism \\
\hline glutamate & metabolism & \\
\hline
\end{tabular}




\begin{tabular}{|c|c|c|c|}
\hline $\mathrm{p}$ value & 0.013036 & 0.016826 & 0.039418 \\
\hline Impact & 1 & 0.24194 & 0.076410 \\
\hline $\begin{array}{l}\text { constituent } \\
\text { structures }\end{array}$ & (lll & (N) & (1) \\
\hline Pathway & http://www.kegg.jp/path & http://www. kegg. jp/ & http://www. kegg. jp/pathway/rno00230 \\
\hline links & $\begin{array}{l}\text { way/rno00471+C00025 } \\
+\mathrm{C} 00026\end{array}$ & $\begin{array}{l}\text { pathway/rno00340+C0 } \\
0025+\mathrm{C} 00135+\mathrm{C} 01152\end{array}$ & $\begin{array}{l}+\mathrm{C} 00020+\mathrm{C} 00212+\mathrm{C} 00294+\mathrm{C} 00242+\mathrm{C} 00387 \\
+\mathrm{C} 00147\end{array}$ \\
\hline
\end{tabular}

\section{Discussion}

221 Metabolomics is an emerging technology for different metabolic pathways of the products

222 by stimulating or disturbing the differences in metabolites displayed by organisms. It can

223 qualitatively, quantitatively and systematically study endogenous small molecules (including

224 blood, urine, and the overall and dynamic laws of tissue fluids) and is widely used in the early

225 diagnosis of diseases, biomarker discovery, pathogenesis research and pharmacological

226 mechanism research. This technology has obvious integrity and dynamic characteristics consistent

227 with the overall theory of traditional Chinese medicine. There is also great potential in explaining

228 the pathogenesis of diseases [8]. Combining pattern recognition and other informatics methods can 
229 be used to analyze the changes in metabolic products caused by physiological and pathological

230 stimuli of organisms and genetic factors. PCA technology can reduce data dimensions and

231 maintain original data, which can improve the detection of abnormal data. It cannot, however,

232 ignore intragroup errors and eliminate random errors that are irrelevant to the content of the study,

233 while PLS-DA and OPLS-DA can maximize the specified sample differences in the analysis,

234 making it easier to find differential metabolic profiles. MetPA can find interfering metabolic

235 pathways and establish a regression model through the commonly used data processing methods

236 of topological analysis [9] and make a discriminant analysis of the regression results [10]. OPLS-

237 DA makes the difference between groups on the basis of PLS-DA useful to help analyze related

238 metabolic pathways [11]. Therefore, the combination of these four methods is a powerful tool for

239 determining disease biomarkers. Comparing the expression of urine metabolites from the level of

240 metabolomics and determining differential metabolites and metabolic pathways can provide an

241 objective basis for the clinical diagnosis of CAG.

242 Liquid chromatography-mass spectrometry (LC-MS) is based on the principle of high-

243 performance liquid chromatography (HPLC) adsorption combined with the analysis technique of

244 the mass-to-charge ratio of charged particles. It is used to determine the mass of particles and

245 determine the elemental composition of samples or molecules to elucidate the chemical structure

246 of the molecule. Compared with other chromatographic methods, the LC-MS sample preparation

247 time is short, has high selectivity, and is not affected by chromatographic resolution. It can carry

248 out the structural analysis of compounds to identify known and unknown compounds and can 
249 quickly identify and elute metabolites. Analysis, through limited instrument optimization, easily

250 obtains quantitative and qualitative data [12].

251 In this study, the animal model of chronic atrophic gastritis was mainly prepared by MNNG

252 combined with ammonia-free drinking water and hunger and satiety. The process of MNNG

253 alkylating the DNA bases does not depend on enzymatic metabolism and can directly penetrate

254 into the pylorus and stomach to cause canceration [13]. Alcohol can trigger acute ischemic damage

255 to the gastric mucosa, causing damaged genes to fail to recover over time, which may be an

256 important factor for initiating oncogenes [14]. Moreover, alcohol can accelerate the dissolution of

257 MNNG and increase the mutation rate. Ammonia can simulate toxic damage to the stomach after

258 Helicobacter pylori infection and maintain acute inflammation of the gastric mucosa $[15,16]$.

259 Ranitidine hydrochloride can inhibit gastric acid secretion, but hunger and satiety are the fusion of

260 spleen and stomach damage. CAG is a complex disease with multiple factors and multiple genes.

261 Compound factor modeling can simulate human disease characteristics to a greater extent and is

262 currently the most widely used and most mature CAG model application.

263 Through PCA, PLS-DA and OPLS-DA LC-MS diversified analysis, using statistics,

264 bioinformatics, chemometrics and other methods to analyze and compare the differential

265 metabolites, the model group and the blank group of rat urine had significant metabolic

266 differences. A total of 68 different metabolites were screened, and 23 metabolic disturbance

267 pathways were predicted. The metabolic pathways can regulate the growth, differentiation,

268 apoptosis and the immune system of tumor cells [17]. The statistically significant metabolic 
269 pathways are D-glutamine and D-glutamic acid metabolism, histidine metabolism, and purine

270 metabolism. Among the metabolic pathways, the significantly different metabolites included L-

271 glutamic acid and 10 different products, including ketoglutaric acid, histidine, 3-methyl-L-

272 histidine, adenosine monophosphate, adenosine, adenine, hypoxanthine, guanosine and guanine.

273 L-Glutamic acid, which is in the metabolic pathway of D-glutamine and D-glutamic acid,

274 plays an important role in protein metabolism in organisms. Studies have found that L-glutamic

275 acid can inhibit cerebral cortex, hippocampal, gastric cancer cell and neural stem cell proliferation

276 and differentiation and induce apoptosis $[18,19]$. Decreased glutamate expression levels will cause

277 digestive system diseases. Based on this performance, L-glutamic acid is a commonly used

278 therapeutic drug for the digestive system, especially gastric cancer and pancreatic cancer.

279 Penicillin can induce the generation of glutamic acid and upregulate cycle-related expression genes

280 and sugar degradation process of glucose to 2-oxoglutaric acid [20].

281 In the histidine metabolism pathway, 3-methyl-L-histidine, histidine, and L-glutamic acid

282 play the role of substrate, intermediate, and product, respectively, and protein nutrition comes from

283 the content of 3-methyl-L-histidine. Each of these compounds are effective indicators of histidine

284 metabolic status [21]. Studies have confirmed that histidine can inhibit the proliferation and

285 migration of lung cancer cells, thereby exerting an antitumor effect [22]. Histamine formed after

286 the decarboxylation of histidine can relax blood vessels and is associated with inflammation. In

287 gastritis and in the duodenum, the reaction in ulcers is sensitive. Currently, histidine is mostly used

288 for the treatment of reducing gastric acid, relieving gastrointestinal pain and as a blood pressure 
treatment. L-Glutamic acid is formed after a series of processes, such as phosphoester and propionic

acid formation, and its antagonists can reverse the abnormal expression of mGlu R5 and PSD-95 in

the striatum of LID rats [23].

Purine metabolism provides cells with the necessary energy and cofactors to promote the

293 growth and proliferation of cells. The most common disease with purine dysfunction is gout, and

294 purine metabolism and its metabolites include adenosine monophosphate, adenosine, adenine, and,

295 at times, the abnormal expression of xanthine, guanosine and guanine will promote the occurrence

296 of gastric cancer [24]. The decomposition of purine nucleotides will promote the

297 dephosphorylation of inosine or guanylic acid and generate inosine or guanosine, which can

298 decompose into xanthine or guanine. The CN-II enzyme is highly expressed in tumor cells [25].

299 Studies have shown that purine nucleotides are essential for metabolic functions. Hypoxanthine,

300 guanine phosphoribosyl transferase and other related purines can affect hematopoietic stem cell

301 cycle progression, proliferation kinetics and changes in mitochondrial membrane potential [26].

\section{Conclusions}

Metabolomics is an important technical means for studying the pathogenesis of diseases.

305 This experiment is the first to use LC-MS metabolomics to study the pathogenesis of CAG from

306 the perspective of urine metabolites. Fromm the method (PCA) and supervised analysis method

307 (PLS-DA and OPLS-DA), differential metabolites of the model group and the control group were 
309 glutamine metabolism with L-glutamic acid, 2-ketoglutarate in the D-glutamic acid metabolism

310 pathway, 3-methyl-L-histidine, histidine, L-glutamic acid and purine in the histidine metabolism

311 pathway. Adenosine monophosphate, adenosine, adenine, inosine, guanosine and guanine may be

312 potential biomarkers for the diagnosis of CAG.

313

\section{Acknowledgments}

315 The authors thank Suzhou BioNovoGene Biopharmaceutical Technology Co., Ltd., for their

316 technical assistance.And the Editing and Manuscript Formatting service provided by American

\section{Journal Experts}




\section{References}

320 1. Jiang Y, Qi X, Liu X, Zhang J, Ji J, Zhu Z, et al. Fbxw7 haploinsufficiency loses its

321 protection against DNA damage and accelerates MNU-induced gastric carcinogenesis.

$322 \quad$ Oncotarget. 2017;8: 33444-33456.

323 2. Hao H. Correlation between pathological classification of chronic atrophic gastritis

324 patients and TCM constitution types. Shanxi Provincial Institute of Traditional Chinese

$325 \quad$ Medicine; 2017.

326 3. Wu Q. CKD-MBD related biomarkers based on metabolomics biological screening and research on the toxicity mechanism of bone metabolism. Second Military Medical

4. Hu T. Research on new methods and strategies for the analysis of endogenous lipid compounds based on chromatographic mass spectrometry. Peking Union Medical College; 2018.

5. Kong XR, Yang Y, Li HZ, Liu L, Zhao SM, Liu HY, et al. The effect of different routes and doses of N-methyl-N-nitro N-nitrosoguanidine administration on pathological changes of gastric mucosa in rats. Chin J Integr Tradit West Med Dig. 2015;23: 381-384, 389. 
7. Smith CA, Want EJ, O'Maille G, Abagyan R, Siuzdak G. XCMS: processing mass spectrometry data for metabolite profiling using nonlinear peak alignment, matching, and identification. Anal Chem. 2006;78: 779-787.

8. Zhao F, et al. Liquorice aqueous extract intervenes in the liver metabolomics of Dgalactose-induced aging rats. Chin Herb Med. 2017;48: 3545-3553.

9. Bai Z, Che Y. Application of BP neural network based on PCA in information security. Electron Technol Softw Eng. 2019;206-207. the effect of Danggui-Chuanxiong herb pair on vasoactive substances and adhesion molecules in the serum of acute blood stasis in rats using PLS-DA and multi-attribute comprehensive index methods. Acta Pharm Sin. 2019;54: 1909-1917. visualization. Bioinformatics. 2010;26: 2342-2344.

12. Pitt JJ. Principles and applications of liquid chromatography-mass spectrometry in clinical biochemistry. Clin Biochem Rev. 2009;30: 19-34. of gastric precancerous lesions on inflammatory factors. Hubei J Tradit Chin Med. 2019;41: 3-4.

14. Yuan X. Research progress of rat models of precancerous lesions. J Anhui Tradit Chin Med Coll. 2004;23: 62-64. 
15. Si J, Wu J, Cao Q, Zun JL. Establishment of rat model of chronic atrophic gastritis discussion on the factors of atrophy. Chin J Dig. 2001: 7-10. on chronic gastritis in rats. Jilin Zhong Med. 2009;29: 527-528. mediated immunosuppression. PLoS One. 2011;6: e16195.

18. Gao C, Wang J, Zhou X. Changes of neuronal apoptosis in rat model of kidney yin deficiency induced by L-monosodium glutamate. Chin J Med. 2003: 719-720.

19. Gao S, Wang J. Changes of neural stem cell proliferation and differentiation-related proteins in L-monosodium glutamate rats. J Third Mil Med Univ. 2004: 1524-1526. transcriptome and metabolome of corynebacterium glutamicum during penicillin-induced glutamic acid production. Biotechnol J. 2018;13: 1700612. Sichuan J Physiol Sci. 1988: 56-57. antitumoral effect of the combination of L-histidine methyl esther hydrochloride of anfotericin B with antineoplastics on A549 cells. FASEB J. 2020;34: 1. 
23. Shu H. Experimental study on the effect of metabolic glutamate receptor 5 antagonist

24. Feng X, Ma D, Zhao J, Song Y, Zhu Y, Zhou Q, et al. UHMK1 promotes gastric cancer progression through reprogramming nucleotide metabolism. EMBO J. 2020;39: e102541. 


\section{Supporting information}

387 S1 Fig. Mass Variation Diagram of the Control Group and Model Group.

388 S2 Fig. HE Staining Pathological Sections. A. HE Staining Pathological Sections of Gastric

389 Mucosa in the Control Group $(\times 200)$; 2-B: HE Staining Pathological Sections of Gastric Mucosa

390 in the Model Group $(\times 200)$.

391 S3 Fig. Chromatogram in Total Ion Mode. 3-A: Typical Sample BPC in Positive Ion Mode, 3-B:

392 Typical Sample BPC in Negative Ion Mode.

393 S4 Fig Urine Metabolism Profile of CAG Model Rats in Positive Ion Mode. 4-A: PCA Scores,

394 4-B: PLS-DA Scores, 4-C: OPLS-DA Scores, 4-D: Replacement Test of the CAG Model Urine

395 Fit Model in Positive Ion Mode.

396 S5 Fig Urine Metabolism Profile of CAG Model Rats in Negative Ion Mode. 5-A: PCA Scores,

397 5-B: PLS-DA Scores, 5-C: OPLS-DA Scores, 5-D: Replacement Test of the CAG Model Urine

398 Fit Model in Negative Ion Mode.

399 S6 Fig Heat Map of the Differential Metabolites. A: Heat Map of the Differential Metabolites in

400 CAG Rats; 6-B: Correlation Heat Map of Differential Metabolites in CAG Rats.

401 S7 Fig Metabolic Pathways of CAG Rat Metabolites Mapped to KEGG. 